\title{
Enhancement in Leach Protocol for Wireless Sensor Networks
}

\author{
Priyanka Bawa \\ Department of Electronics and \\ Communication ,Amity School \\ of Engineering and \\ Technology,AUUP,India
}

\author{
Bhupendra Singh \\ Department of Electronics and \\ Communication ,Amity School \\ of Engineering and \\ Technology,AUUP,India
}

\author{
Vinod Shokeen \\ Department of Electronics and \\ Communication ,Amity School \\ of Engineering and \\ Technology,AUUP,India
}

\begin{abstract}
A wireless sensor network (WSN) consists of spatially distributed autonomous sensors to monitor physical or environmental conditions, such as temperature, sound, pressure, etc. and to cooperatively pass their data through the network to a Base station or sink which communicates the data further for processing. The sensor nodes have limited battery power sources and bandwidth. LEACH protocol is one of the clustering routing protocols in wireless sensor networks which is able to distribute energy dissipation evenly throughout the sensors. This paper focuses on even distribution of the energy load among the sensor nodes in order to overcome the problem of overly utilized sensor nodes that will run out of energy as compared to other sensor nodes. The proposed protocol is an enhancement of Leach which selects the top ten percent of the total sensor nodes which have highest residual energies. The proposed protocol will optimize the energy of the WSN and increase the network lifetime as compared to Leach protocol. There are two phases of the protocol i.e. setup phase and steady phase.In this work, MatLab 7.9 version has been used for the simulation work.
\end{abstract}

\section{General Terms}

Wireless Sensor Networks, Communication.

\section{Keywords}

Wireless sensor networks, base station, Clustering, Leach protocol, network lifetime.

\section{INTRODUCTION}

The emerging field of wireless sensor networks combines sensing, computation, and communication into a single tiny device. These sensor nodes are scattered in an unattended environment(i.e. sensing field) to sense the physical world. The sensed data can be collected by a few sink nodes which have accesses to infrastructured networks like the Internet. The most straightforward application of wireless sensor network technology is to monitor remote environments for low frequency data trends. [1]. A wireless sensor network system usually includes sensor nodes, sink node and management node. A large number of sensor nodes are deployed in the monitored area, constituting a network through the way of self-organization. The data monitored by sensor nodes is transmitted along other nodes one by one, that will reach the sink node after a multi-hop routing and finally reach the management node through the wired and (or) wireless Internet[2]. The energy, the ability of signal process, storage capacity and communication capability of sensor nodes are very limited.
A primary design goal for wireless sensor networks is to use the energy efficiently[3].Cluster-based routing algorithm has a better

energy utilization rate compared with non-cluster routing algorithm[4].

In clustering routing[3][7] the nodes are grouped into clusters and one node of the cluster which is the cluster head sends all gathered data from the nodes in its cluster to the base station. Hence it uses the information aggregation mechanism in the cluster head to reduce the amount of data transmission, thereby, reduce the energy dissipation in communication and in turn achieve the purpose of saving energy of the sensor nodes. In the clustering routing algorithms for wireless networks, LEACH (low-energy adaptive clustering hierarchy)[4][5] is well-known because it is simple and efficient. LEACH divides the whole network into several clusters, and the run time of network is broken into many rounds. In each round, the nodes in a cluster compete with each other to be cluster head according to a predefined criterion. In LEACH protocol, all the sensor nodes have the same probability to be a cluster head.This feature leads to a balanced distribution of the energy consumption to all nodes and makes it possible to have a longer lifetime for the entire network.

Some works have been conducted on the performance and enhancement of LEACH protocol[1][6]. This paper proposes an enhanced version of Leach Protocol which aims at prolonging the lifetime of WSN by changing the criterion of selecting cluster heads.

\section{LEACH[4]}

All LEACH is the first hierarchical protocol in WSN. LEACH is an adaptive clustering routing protocol proposed by Wendi B. Heinzelman. In many later literatures, it has been considered as the benchmark for other protocols. It has some distinctive characteristics like self-reconfiguration, adjustment of communication range according to distance, schedule of data transmission of individual nodes etc. In LEACH, the nodes organize themselves into local clusters, with one node acting as the cluster-head. All non-cluster-head nodes must transmit their data to the cluster-head, while the cluster-head node must receive data from all the cluster members, perform signal processing functions on the data (e.g., data aggregation), and transmit data to the remote base station. Therefore, being a cluster-head node is much more energyintensive than being a non-cluster-head node. In the scenario where all nodes are energy-limited, if the cluster-heads were chosen a priori and fixed throughout the system lifetime, as in a static clustering algorithm, the cluster-head sensor nodes 
would quickly use up their limited energy. Once the clusterhead runs out of energy, it is no longer operational.

The operation of LEACH is broken up into rounds, where each round begins with a setup phase, when the clusters are organized, followed by a steady-state phase, when data transfers to the base station occur. In order to minimize overhead, the steady-state phase is long compared to the setup phase. Initially, when clusters are being created, each node decides whether or not to become a cluster-head for the current round. This decision is based on the suggested percentage of cluster heads for the network (determined a priori) and the number of times the node has been a clusterhead so far. This decision is made by the node $\mathrm{n}$ choosing a random number between 0 and 1 . If the number is less than a threshold $\mathrm{T}(\mathrm{n})$, the node becomes a cluster-head for the current round. The threshold is set as:

$$
T(n)= \begin{cases}\frac{P}{1-P *\left(\operatorname{rmod} \frac{1}{P}\right)} & \text { if } \mathrm{n} \in \mathrm{G} \\ 0 & \text { otherwise }\end{cases}
$$

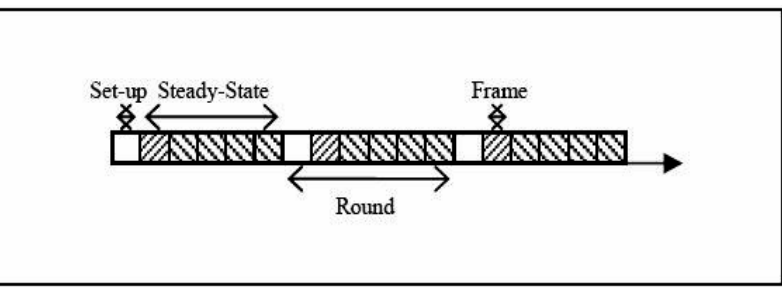

Figure 1: LEACH protocol phases

\section{DESCRIPTION OF THE PROPOSED PROTOCOL}

The proposed protocol is based on distributed clustering. In this protocol, all the nodes which are deployed randomly in the required environment to be monitored will be capable of communicating to every other node in the scenario. Every node will advertise its energy levels to all other nodes. The energy level of every node will be checked individually by each node and the nodes which are having energy more than the average energy level of the network are nominated for Cluster Head. The nodes which are at the top ten levels will be selected as Cluster Head initially. These Cluster Heads will advertise their selection to every other node in the network. Those nodes which are not capable of becoming the Cluster Head will wait for the advertisement made by the $\mathrm{CH}$. These nodes will send a join request to the $\mathrm{CH}$ which is nearest to them on the basis of received signal strength. The Cluster Head will accept the cluster members and will prepare a TDMA schedule so that each node can communicate to $\mathrm{CH}$ without any collision. This TDMA schedule will be sent to the cluster members. Since the nodes will always have data to be send to $\mathrm{CH}$, there are chances that these data may be correlated. The data collected at Cluster Head after the completion of one TDMA Cycle will be fused and aggregated. Now the communication between $\mathrm{CH}$ and the Base station will take place with the help of Direct Spread Spectrum so that no collision of data takes place. One by one the $\mathrm{CH}$ will be sending the aggregated data to the Base station for monitoring. After one round the process will be repeated again.

\section{WORK FLOW OF THE PROPOSED PROTOCOL}

This section describes diagrammatically the Phases of the proposed protocol. In the Phase 1 i.e. Set Up Phase, every node will advertise its energy level to the other nodes in the network. An average energy level of the network will be calculated in every node. All those nodes which are having energy level greater than or equal to the average energy level will be nominated for Cluster Head. The energy levels will be sorted in descending order by every live node in the network. Since all the nodes are identified by their Identification number, those nodes which are in the top ten level of the energy level will match their identification. After getting selected as cluster head, the $\mathrm{CH}$ nodes will advertise their selection and the rest of the non Cluster head nodes will send join request based on the received signal strength of the advertisement of $\mathrm{CH}$ nodes to the nearest $\mathrm{CH}$. The Cluster Head will accept their request and will prepare a TDMA slot and the member nodes will send data accordingly. This data will be collected at Cluster Head and it will aggregate that that and will transmit this data to the Base station for monitoring.

\subsection{SETUP PHASE}

The setup phase consists of three parts i.e. Cluster Head Election, Cluster Formation and Schedule Creation. In the Cluster Head Election, nodes having energy level greater than the average energy level of network are nominated and then elected as Cluster Head on the basis of larger energy level. In the Cluster Formation, Non Cluster Head nodes join cluster head based on received signal strength and form the cluster. In the Schedule Creation, the Cluster Head prepares a TDMA schedule for its cluster members for data transmission. A node in the network checks whether it is selected as Cluster Head or not. If the node is not selected as Cluster Head, It will wait for the announcement to be made by the Cluster Head nodes. If the node is selected as Cluster Head, It will advertise its selection to the other nodes in the network so that the Non Cluster Head nodes can join it to become the cluster members. The Cluster Head will wait for the Join Request to be made by the non cluster heads for a time period known a prior. Non cluster head nodes will send Join Request to the Cluster Head which is nearest to them based on the received signal strength of the advertisement made by the Cluster Head nodes. The Cluster Head after getting the responses from the nodes will then prepare a TDMA schedule for its members and will again advertise this schedule only to its cluster members. This schedule will be saved by the cluster members and will send data on the basis of the allotted TDMA slot. After this the steady phase comes into play and after the completion of a round this process will be repeated again. In this way a complete round takes place. 


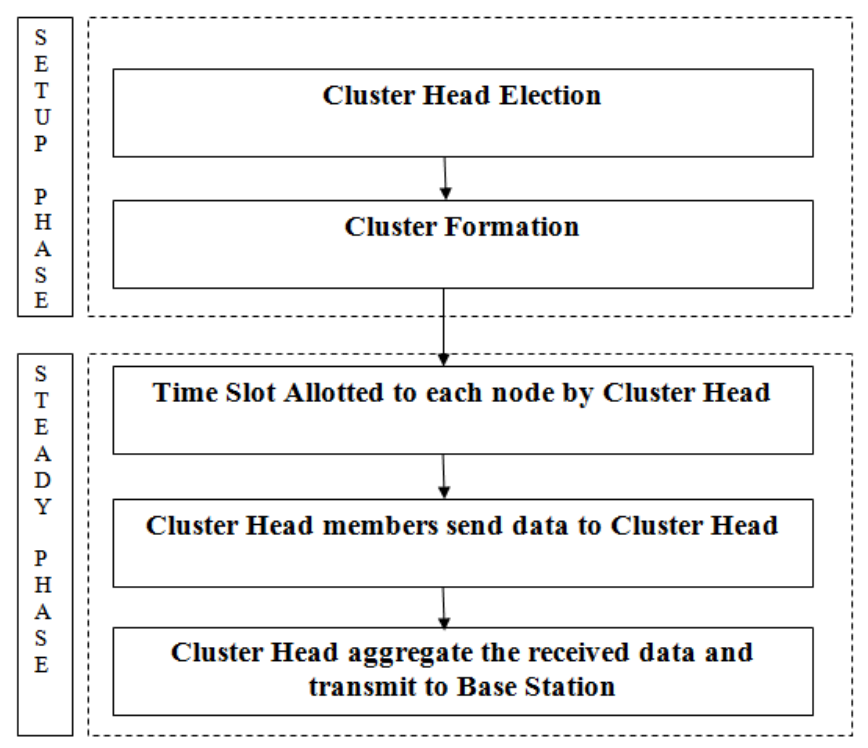

Figure 2: Workflow of the Proposed Protocol

\subsubsection{Cluster Head Election}

In this phase, every node in the network advertises its energy level to the every other node of the network. After this advertisement every node possesses the energy level of each and every node of the network. Now every node will arrange the received energy level of other nodes in the ascending order. The average energy of the network can be calculated as follows:

$$
\text { Average Energy }=\frac{\sum_{i}^{n} \text { Energy of each node } i}{\text { Total number of alive nodes }}
$$

Those nodes whose energy level is less than the average energy level of the network will be discarded from the Cluster head nomination criteria. Now every node will match its ID with the top ten nodes having the highest energy level. If the ID matches, the node is selected as the Cluster Head. If the ID does not match, the node will wait for the Cluster Head announcements.

\subsubsection{Cluster Formation}

After getting selected as the Cluster Head, now the Cluster Heads will let the other nodes in the network know that they have chosen the role of Cluster Head for the current round. Now the Cluster head will advertise a Join Request message to all other nodes using a non persistent carrier sense multiple access (CSMA) MAC protocol [9]. This message consists of the Node ID and the Header of this message will be different from normal data messages. Each and every node which is non Cluster Heads will wait for this Join Request Message. The Cluster head will wait for the response for a time period known a prior. Every node will receive this announcement. Now the Non Cluster Head nodes will arrange the received announcements in the decreasing order of the received signal strength. Received Signal Strength is chosen because larger the received signal strength, more the node closer to it unless and until there is an obstacle in between the nodes. The nodes will check which Cluster Head is nearer to it i.e. whose received signal strength is strongest. And will select that
Cluster Head. Now the node needs to inform the Cluster Head about its selection. The node will send a Join Response message which will consist of Node ID and a header differentiating it from data messages using a non persistent carrier sense multiple access (CSMA) MAC protocol back to the closest Cluster Head. The Cluster Head will accept the Response and add the node as its cluster member.

\subsubsection{Schedule Creation}

Since the Cluster Head also acts as Local data collection center, it will control and coordinate the data transmission between the cluster members. Now all the Cluster Heads will advertise their Cluster member information in between the Cluster Head nodes. The Cluster Head with the largest number of cluster member will set up a time period for the TDMA one cycle. For example, if one of the Cluster Head consists of 20 members and other Cluster Head consist of 10 members then the former Cluster Head will provide one second time allotment for each cluster member and the later Cluster Head will allot two seconds slot for each cluster member. This is done so that the TDMA cycle of each and every Cluster Head should complete at the same time. After the preparation of TDMA schedule by the Cluster Head, the Cluster Head sends this schedule to all its cluster members. Now the cluster members will send their data on the time slot allotted to them. Suppose there are 15 nodes in a cluster. After transmitting the data to the cluster Head, the node will go in sleep mode with its sensor active. Hence the energy of the node can be saved in sleep mode

\subsection{STEADY PHASE}

This phase is the second phase of the proposed protocol. The steady phase consists of Data collection \& Aggregation along with Data Transmission

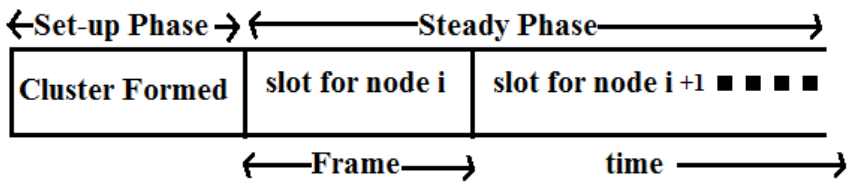

Figure3: steady phase

In Data collection, the cluster head gather data from every cluster member and fuse the data so that no redundancy left. In the data transmission, the Cluster Heads transmit the data one by one to the Base Station for monitoring.

\subsubsection{Data Collection and Aggregation}

Data Collection and Aggregation is important part of the Wireless Sensor Network. As the wireless sensor network is bounded by its limited energy supply, therefore emphasis should be made to transmit as less data as possible because transmission consumes most of the power of the wireless sensor network. Aggregation of data is required because if two homogeneous sensors are placed in a same geographical area, then the data sensed by them will be correlated or redundant. Hence Aggregation of data will reduce redundancy thereby decreasing the energy consumption of the network.

The Data collection operation is broken into frames where member nodes send their data to the cluster head at most once per frame during their allotted TDMA slot. The time duration 
for each slot is fixed where the node sends data to the Cluster Head. The data collected is then fused or aggregated by the Cluster Head so that less number of bits of data is to be sending to the Base Station. The time slot depends on the number of cluster members. It is presumed that the nodes are all time synchronized and the setup phase of the network is initiated at the same time. This is done so that each round of the clusters of the network should get completed at the same time.

\subsubsection{Data Transmission}

In this the data collected at Cluster Head is send to the Base Station. To reduce the energy dissipation, each node of the network uses power control mechanism. In this mechanism all nodes in the network can control their transmission and reception power as per the requirement. Suppose the maximum transmission range of a node is $200 \mathrm{~m}$, and the distance between the node and its Cluster Head is $35 \mathrm{~m}$, now if the node is transmitting the data with its maximum range, then it is dissipating its power uselessly in the environment. So the nodes can adjust the transmission power level as needed i.e. for above example the node and the Cluster head will be transmitting the data with a maximum transmission power range of $40 \mathrm{~m}$ so that the energy could be saved.

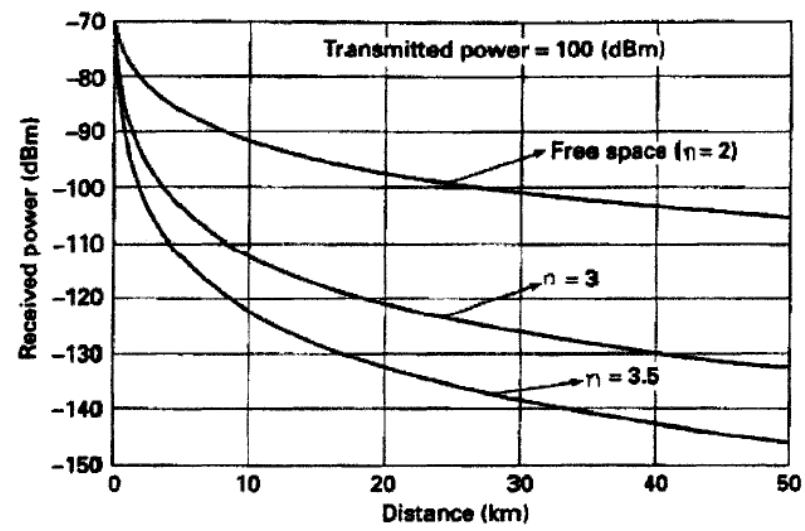

Figure 4:Wireless Communication Radio Model

The data to be sent to Base Station from Cluster Head is done by using fixed spreading code and CSMA. Whenever the Cluster Head has data to be sent to Base Station, it will first sense the channel to ensure that no other Cluster Head is sending the data to the Base Station. If the channel is busy or other Cluster Head are transmitting the data, then the Cluster Head will wait for a time period and then again sense the channel. Now if the channel is free the Cluster Head will send the data using DSSS. DSSS is used for data transmission because the number of cluster heads may vary after some rounds. Other channelization technique like FDMA can be used but it is harder to allot frequency dynamically. The drawback of DSSS is that it requires tight synchronization timing.

\section{ENERGY CONSUMPTION MODEL}

Wireless Communication Energy model consists of five major blocks: transmitter's digital physical layer (PHY) and Medium Access Control (MAC) functions, transmitter's RF Circuitry including the Phase Locked Loop and Voltage Control Oscillator (VCO), power amplifier, receiver's RF circuitry and receiver's digital functions [10].In the proposed protocol, First Order Radio model is assumed [4]. In this model the radio dissipates $\mathrm{E}_{\text {elec }}=50 \mathrm{~nJ} / \mathrm{bit}$ to run the transmitter or receiver circuitry and $\mathrm{E}_{\mathrm{amp}}=100 \mathrm{pJ} / \mathrm{bit} / \mathrm{m}^{2}$ for the transmit amplifier to achieve an acceptable Eb/No. These parameters are slightly better than the current state-of-the-art in radio design

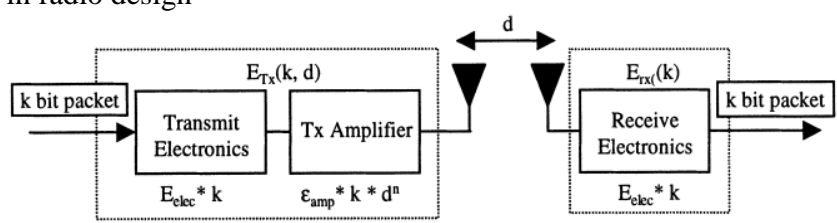

Figure 5:First Order Radio Model[4]

We also assume an $r$ energy loss due to channel transmission. Thus, to transmit a k-bit message a distance $d$ using our radio model, the radio expends:

$\mathrm{E}_{\mathrm{TX}}(\mathrm{k}, \mathrm{d})=\mathrm{E}_{\mathrm{TX}}-\operatorname{elec}(\mathrm{k})$
$\mathrm{E}_{\mathrm{TX}}(\mathrm{k}, \mathrm{d})=E_{\mathrm{TX}}-\operatorname{amp}(\mathrm{k}, \mathrm{d})$
elec

Table 1:Radio Model's Operations energy dissipation[4]

\begin{tabular}{|l|c|}
\hline Operation & Energy Dissipated \\
\hline \hline Transmitter Electronics $\left(E_{T x-e l e c}\right)$ & \\
Receiver Electronics $\left(E_{R x-e l e c}\right)$ & $50 \mathrm{~nJ} / \mathrm{bit}$ \\
$\left(E_{T x-e l e c}=E_{R x-e l e c}=E_{\text {elec }}\right)$ & \\
\hline Transmit Amplifier $\left(\epsilon_{a m p}\right)$ & $100 \mathrm{pJ} / \mathrm{bit} / \mathrm{m}^{2}$ \\
\hline
\end{tabular}

And to receive this message, the radio expends:

$$
\begin{aligned}
& \mathrm{E}_{\mathrm{RX}}(\mathrm{k})=\mathrm{E}_{\mathrm{RX}}-\operatorname{elec}(\mathrm{k}) \\
& \mathrm{E}_{\mathrm{RX}}(\mathrm{k})=E_{\text {elec }} * \mathrm{k}
\end{aligned}
$$

For these parameters, receiving a message is also costlier, hence the protocol should try to minimize not only the transmitting distance but also the number of transmit and receive operations. In the proposed protocol, an assumption is made such that the energy dissipated in transmitting a message from a Node A to Node B is equal to the energy dissipated in transmitting a message from Node B to Node A. In the proposed protocol it is also assumed that sensor node has data all the time to transmit to the Cluster Head.

\section{SIMULATION AND RESULTS}

MatLab is used as a simulation platform.With MatLab, it is easier to generate a GUI to show the scenario of the Wireless Sensor Network. There are many functions provided in the MatLab which makes the simulation work easier. MatLab provides quicker configuring the parameters. The different plots can be made in the same simulation with the help of MatLab. Simulation in MatLab is quicker and less complex. There are many protocols which are already implemented in MatLab. Therefore the proposed protocol is implemented in MatLab Version 7.9 


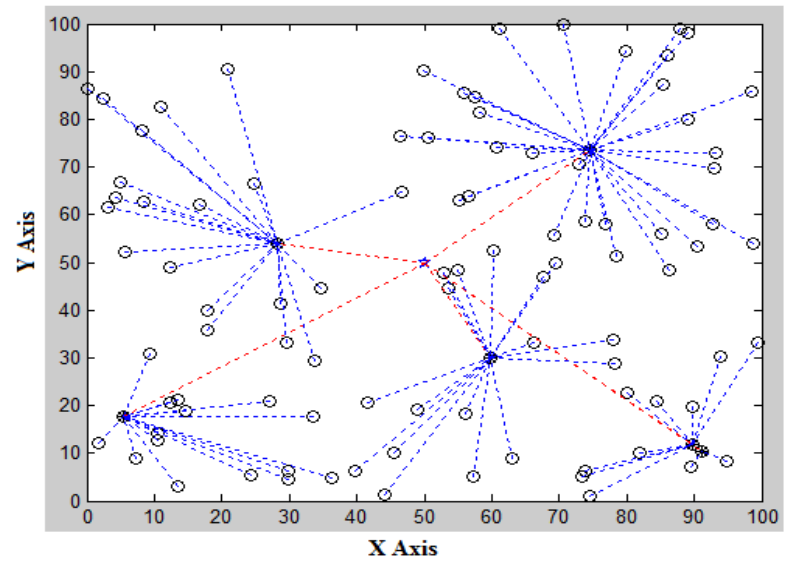

Figure 6: Scenario of Proposed Work Simulation with Base Station at $(\mathbf{5 0 , 5 0 )}$

\subsection{Parameters Description}

The simulation is carried out in a network grid of $100 \mathrm{mX} 100 \mathrm{~m}$. There are two scenarios where the position of the Base station is varied. In one scenario, the location of the Base station is $(50,50)$ and in another scenario, the Base station is at $(175,50)$. The numbers of nodes taken are 100 . But for the extension of protocol the number of nodes is changed to 200 to find out the proposed protocol behaviour. The percentage of Cluster Head is chosen $10 \%$ of the live nodes in the wireless sensor network. This has been chosen $10 \%$ by the experimental analysis as shown in figure 6 . The energy levels of each node are also varied in both the scenario. The energy levels are set as $0.25 \mathrm{~J}, 0.50 \mathrm{~J}$, and $1 \mathrm{~J}$. In some of the experiments the energy level is chosen randomly for every sensor node in the network. It is done so that the proposed protocol should ensure it is more efficient than the LEACH protocol [4]. In some experiments the energy levels are set in multiples of 5 to 20 Joule to know about the lifetime pattern of the network as it will reveal that the network lifetime increases proportionately with the energy of the wireless sensor network. In this model the radio dissipates $E_{\text {elec }}=50 \mathrm{~nJ} / \mathrm{bit}$ to run the transmitter or receiver circuitry and $\mathrm{E}_{\mathrm{amp}}=100 \mathrm{pJ} / \mathrm{bit} / \mathrm{m}^{2}$ for the transmit amplifier. $\mathrm{E}_{\mathrm{DA}}=5 \mathrm{~nJ} / \mathrm{bit} / \mathrm{signal}$ is the Energy required for data aggregation. $€_{\mathrm{fs}}$ is the energy dissipated for free space and $€_{\mathrm{mp}}$ is the energy dissipated for multi path fading in wireless sensor network. Different topologies are generated randomly for simulation. Each simulation result shows the average of independent experiments.

Table 2:Simulation Parameters for Proposed Protocol

\begin{tabular}{|l|l|}
\hline Parameters & Values \\
\hline Network Grid & From $(0,0)$ to $(100,100)$ \\
\hline Number of Nodes & 100 \\
\hline Base Station Location & $(175,50) \mathrm{m} /(50,50) \mathrm{m}$ \\
\hline Initial Energy Eo & $0.25 \mathrm{~J} / 0.50 \mathrm{~J} / 1 \mathrm{~J}$ \\
\hline$€_{\mathrm{fs}}$ & $10 \mathrm{pJ} / \mathrm{bit}^{\mathrm{m}} \mathrm{m}^{4}$ \\
\hline$€_{\mathrm{mp}}$ & $0.0013 \mathrm{pJ} / \mathrm{bit} / \mathrm{m}^{4}$ \\
\hline $\mathrm{E}_{\mathrm{elec}}$ & $50 \mathrm{~nJ} / \mathrm{bit}$ \\
\hline $\mathrm{E}_{\mathrm{DA}}$ & $5 \mathrm{~nJ} / \mathrm{bit} / \mathrm{signal}$ \\
\hline
\end{tabular}

Figure 7:Cluster Head Percentage Selection
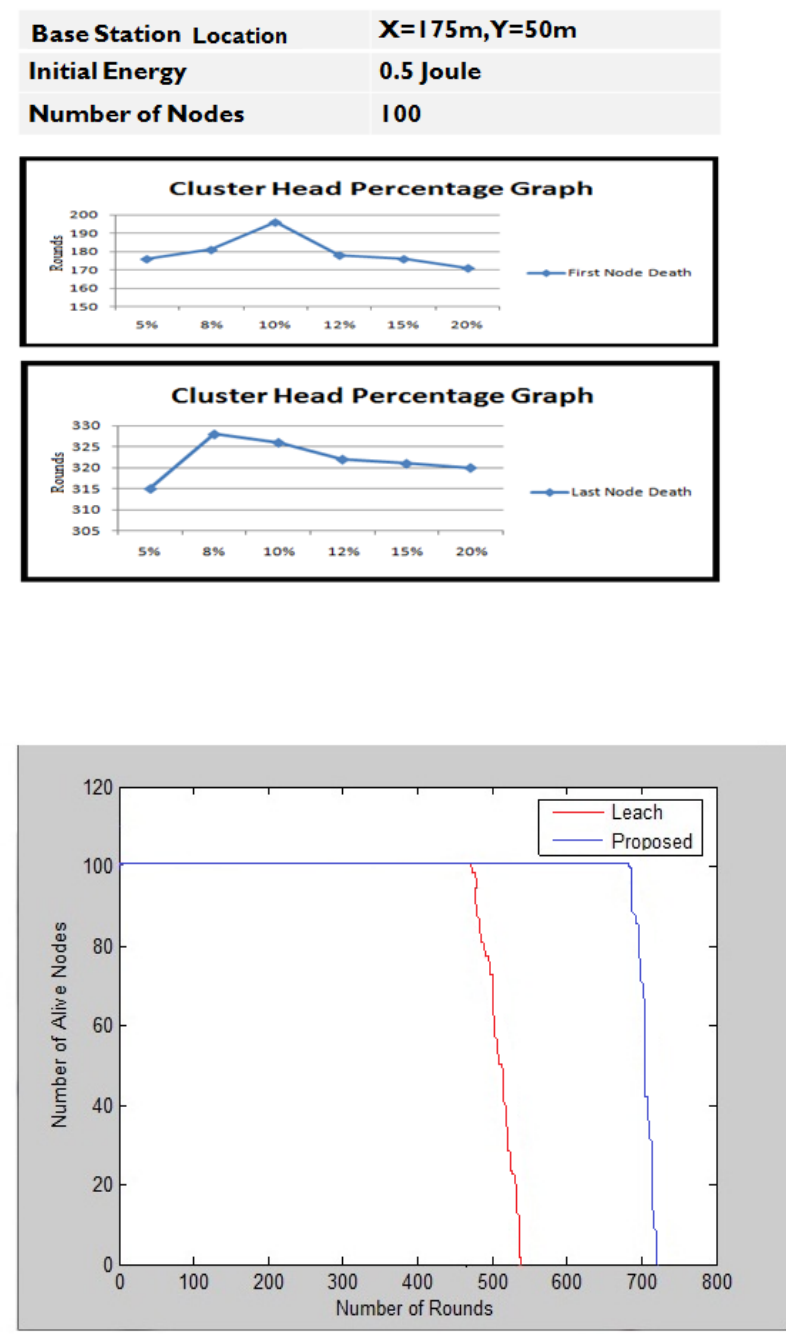

Figure 8: Plot of Alive nodes vs. Round $(x=175 m$, $\left.\mathrm{y}=50 \mathrm{~m}, \mathrm{E}_{\mathrm{O}}=\mathbf{0 . 5 \mathrm { J }}\right)$

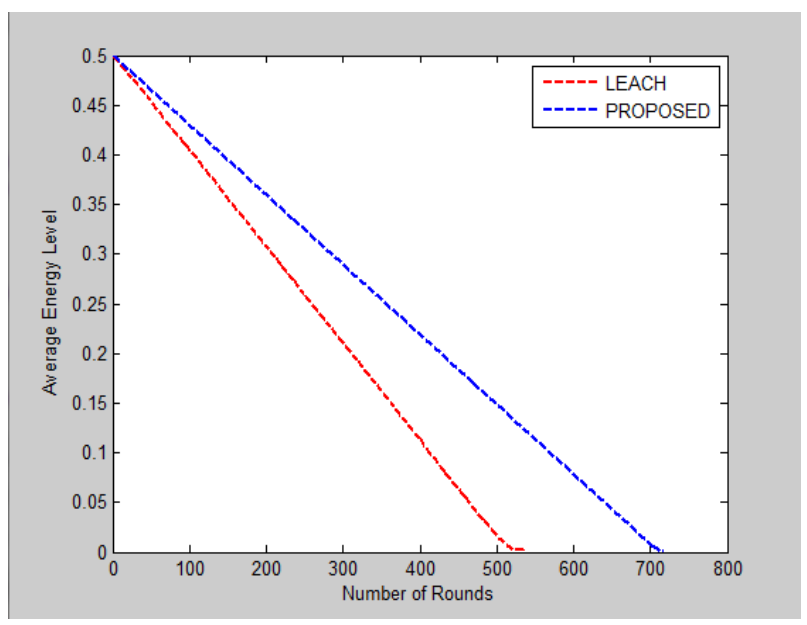

Figure 9: Plot of Average Energy Level of nodes vs. Round $\left(x=175 \mathrm{~m}, y=50 \mathrm{~m}, \mathrm{E}_{0}=0.5 \mathrm{~J}\right)$ 
From figure 8, we can see that the death of first node in LEACH is quicker as compared to the proposed protocol. Hence the lifetime of the network is increased in all the cases.

Figure 9 depicts the average energy level of protocols in terms of rounds. We can analyze that the average energy of LEACH protocol is falling rapidly as compared to the proposed protocol.

\section{CONCLUSION}

The proposed protocol selects ten percent of the live node as cluster head for the cluster formation. These cluster heads possesses the highest energy levels in the wireless sensor network. This is done so because the cluster head utilises more energy as compared to the other nodes in the network as it receives the data packet send by its cluster member, it aggregates and fuses the gathered data and send the data to the Base Station. Thus the proposed protocol improves the network lifetime and hence is more energy efficient than Leach protocol.

\section{REFERENCES}

[1] D. Culler, D. Estrin and M. Srivastava, "Guest editors' introduction: Overview of sensor networks," Computer, vol. 37, no. 8, pp. 41-49, Aug. 2004.

[2] J. Hu, Y. Jin, and L. Dou, "A Time-based ClusterHeadSelection Algorithm for LEACH," IEEE Symposium on Computers and Communications, 2008,1172-1176. J.D. Yu, K. T. Kim , B. Y. Jung, and H. Y. Youn," "AnEnergy Efficient Chain-Based Clustering Routing Protocol forWireless Sensor Networks,"
Advanced InformationNetworking and Applications Workshops, 2009, 383-384.

[3] N.M.A. Latiff, C.C. Tsimenidis, and B.S. Sharif,"Performance Comparison of Optimization Algorithm for Clustering in Wireless Sensor Networks," IEEE International Conference on Mobile Adhoc and Sensor Systems, 2007, 1-4.

[4] W.R. Heinzelman, A. Chandrakasan, and H. Balakrishnan,"Energy-efficient communication protocol for wireless microsensor networks," Proceedings of the 33rd Hawaii International Conference on System Sciences, 2000, 1-10.

[5] W.R. Heinzelman, A. Chandrakasan, and H. Balakrishnan,"An Application-Specific Protocol Architecture for Wireless Microsensor Networks," IEEE Transactions on Wireless Communications, 2002, 1(4): 662-666.

[6] H. Yang and B. Sikdar, "Optimal Cluster Head Selection in the LEACH Architecture", IEEE International Conference on Performance, Computing, and Communications, 2007, 93-100.

[7] Yoshitsugu Obashi, Tomohiro Kokogawa, Yi Zheng, Huifang Chen, Hiroshi Mineno, and Tadanori Mizuno, "Evaluation of Metadata-Based Data Aggregation Scheme in Clustering Wireless Sensor Networks," Lecture Notes In Artificial Intelligence, vol.4694/2010, pp. 477-483, Sep. 2007, doi:10.1007/978-3-540-74829-8 59. 